\title{
Target recognition based on stratified synthesis strategy
}

\author{
Dangnai Yao*, Hua Chai, and Zhenguo Wang \\ National University of Defense Technology \\ *yaodangnai@sina.com
}

\begin{abstract}
Keywords: target recognition; ballistic missile defense; stratified synthesis strategy; analytic hierarchy process theory.
\end{abstract}

\begin{abstract}
Target recognition is a hot topic in the field of ballistic missile defense. In this paper a stratified synthesis strategy is developed to recognize the real target objectively. Firstly, a hierarchical index system of target characteristic similarity is proposed, which considers the infrared, radar and dynamic-based characteristics respectively. Secondly, the weights of the indices are determined by employing the analytic hierarchy process (AHP) theory. Thirdly, a stratified synthesis strategy is provided to calculate the integrated characteristic similarity of different targets. Simulation example shows the efficiency of the proposed strategy.
\end{abstract}

\section{Introduction}

In the defense against ballistic missiles, if the attacker tries to use decoys to break through the defense system, the defender must rely on effective target recognition to make sure the defense system works successfully, [1]. Thus, target recognition is very important in defense process, lots of efforts have been devoted to obtain satisfied results, [2 7]. Previous researches mostly gave attention on infrared-based recognition [2 4], radar-based recognition [5 7], and so on. However, as these methods concentrated on single physicalcharacteristics, the established system can be cheated by false target. To improve the chances of true-target recognition, it is necessary to explore advanced recognition methods, which consider multiple characteristics of the target.

In this paper the strategy of stratified synthesis $[8,9]$ is introduced to the problem of target recognition. The concept of characteristic similarity is used to measure the similarity degree of a target and true warhead. Different characteristic similarities constitute hierarchical index system which supports the solution of integrated similarity, and the integrated similarity is treated as the metric of target recognition. As the integrated similarity gives a good reference for target characteristics, the probability of true-warhead recognition can be highly improved.

\section{Hierarchical Index System of Target Characteristic Similarity}

In the proposed recognition strategy, we assume that the defender has prior knowledge of different characteristics of true warhead. The recognition process then is to determine the similarity between an arbitrary target and true warhead. We construct a hierarchical index system of target characteristic similarity as shown in Fig. 1. As the physical features of target include infrared feature, radar feature and mechanical feature, the integrated similarity can be divided into three aspects. In each aspect, the similarity can be further divided.

Infrared-Based Similarity. Due to the differences of material and surface temperature between the true warhead and decoy, the infrared-based characteristics between the two turn out to be different.

- The radiant intensity can be obtained by monochrome infrared sensor;

- The temperature can be obtained by bicolor or multicolor sensors;

- The infrared heat image can be obtained by reflecting the infrared radiation on the detector's photosensitive component; 
These features can be treated as reference for infrared-based recognition.

Radar-Based Similarity. Due to the difference of shape, size and material between the true warhead and decoy, the radar-based characteristics between the two is different.

- The RCS feature of the target can be obtained by processing the radar's echo wave;

- The polarization feature can be achieved by comparing the polarized information of radar's echo wave. It should be noticed that such information is determined by the shape, size, structure and orientation of the target;

- When the distance resolution of the radar is largely smaller than the target's size, the target continually fills multiple distance units, which forms a one-dimensional distance image as shown in Figure 2. Further, a two-dimensional image can be obtained by analyzing the Doppler frequency shift of the echo wave in each distance unit.

These features can be used as reference for radar-based recognition.

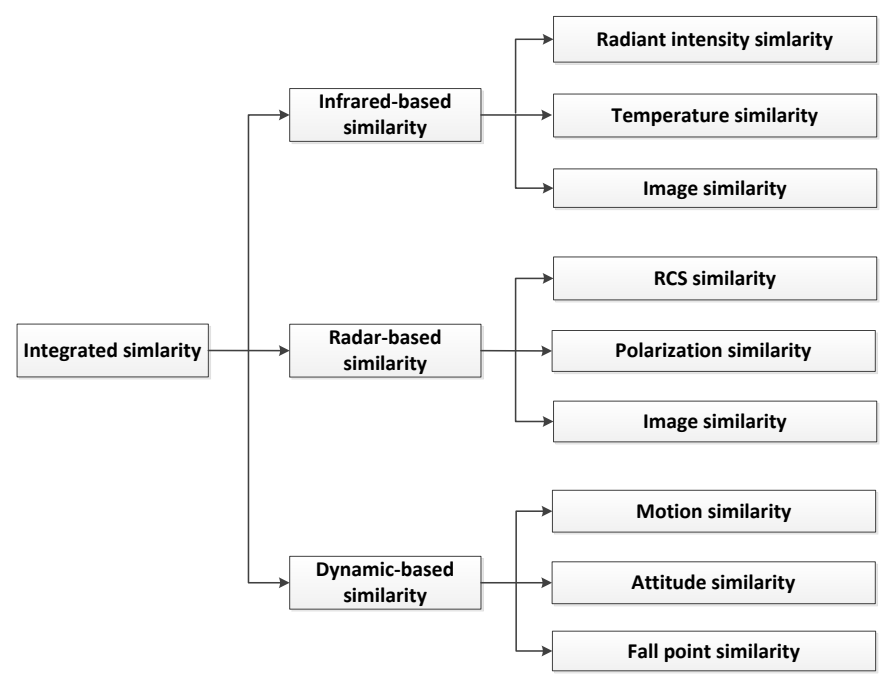

Figure 1. Hierarchical index system of target characteristic similarity

Dynamic-based Similarity. By analyzing the dynamic characteristics of the target, some information can be abstracted for recognition.

- By checking the space distribution of the target group and comparing the relative position among different targets, the result can be a useful reference of recognition between the true warhead and decoy;

- The attitude of the true warhead is more stable than the decoy as the latter may roll around irregularly.

- The fall point can be used to detect the true warhead as the true warhead prefers to be placed near the important place.

Since the similarity index is used to measure the similarity degree between the target and true warhead, they are all in the range of $0 \sim 1$. In this paper, the bottom 9 similarity indices are given by the sensors.

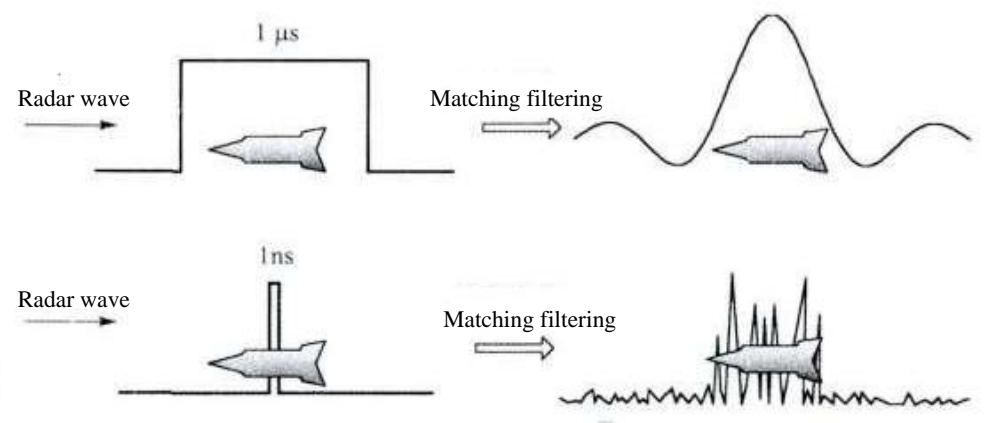

Figure 2. One-dimensional radar's distance image 


\section{Index Weight Determination}

Before the process of synthesis evaluation, the relative importance in the same level should be determined. Present paper tries to use the analytic hierarchy process (AHP) theory $[10,11]$ to calculate the index weight, which is shown in the following subsections.

Judgment Matrix Composition. The AHP theory requires that the evaluator gives the relative importance among different index in the same level. According to Satty's scaling method, the determination matrix can be defined as

$$
A=\left[\begin{array}{cccc}
a_{11} & a_{12} & \cdots & a_{1 m} \\
a_{21} & a_{22} & \cdots & a_{2 m} \\
\cdots & \cdots & \cdots & \cdots \\
a_{m 1} & a_{m 2} & \cdots & a_{m m}
\end{array}\right]
$$

where $a_{i j}$ is the relative importance of index $u_{i}$ compared to $u_{j}$, which is in the range of $1 \sim 9$. Specifically, 1 means the relative importance is the lowest meanwhile 9 means the highest. The value of $a_{i j}$ can be given by the specific specialist. It can be easily learned that the determination matrix in Eq. (1) is reciprocal and consistent.

Index Weight Calculation. In the AHP theory, the calculation of index weight is to calculate the maximum eigenvalue and characteristic root, which is to solve the following function

$$
A \omega=\omega \lambda_{\max }
$$

where $\omega$ is the weight vector.

Consistency Verification. To make sure the reasonability of the determination matrix, its consistency should be evaluated. The metric can be obtained as follows:

$$
C I=\frac{1}{m-1}\left(\lambda_{\max }-m\right)
$$

where $m$ represents the matrix dimension. $C I=0$ shows that the consistency is the best. In the case of large $C I$, its consistency is worse. $C I$ is compared with the random consistency metric $R I$, that is

$$
C R=C I / R I
$$

$C R<0.1$ means the determinant matrix has satisfied consistency thus the selected index vector $\omega$ is reasonable. Otherwise, new determinant matrix is required to satisfy the consistency requirement of $C R$. In Eq (4), the random consistency index is listed in Table 1.

Table 1. Value of Random Consistency Index RI

\begin{tabular}{|c|c|c|c|c|c|c|c|c|c|c|}
\hline $\boldsymbol{m}$ & $\mathbf{1}$ & $\mathbf{2}$ & $\mathbf{3}$ & $\mathbf{4}$ & $\mathbf{5}$ & $\mathbf{6}$ & $\mathbf{7}$ & $\mathbf{8}$ & $\mathbf{9}$ & $\mathbf{1 0}$ \\
\hline $\boldsymbol{R I}$ & 0.0 & 0.0 & 0.58 & 0.90 & 1.12 & 1.24 & 1.32 & 1.41 & 1.45 & 1.49 \\
\hline
\end{tabular}

\section{Stratified Synthesis Strategy}

According to Fig. 1, there are two kinds of weights. One is weights of index; the other is weights of index group.

1) Weights set of index group

Suppose the weight of the $k^{\text {th }}$ index group is $\omega_{k}(k=1,2, \cdots, \mathrm{s})$, the weights set of index group is

$$
\omega=\left[\omega_{1}, \omega_{2}, \cdots, \omega_{s}\right]
$$

2) Weights set of index

Suppose the weight of $j^{\text {th }}$ index in the $k^{\text {th }}$ group is $\omega_{k j}$, its corresponding weight set is

$$
\boldsymbol{\omega}_{k}=\left[\omega_{k 1}, \omega_{k 2}, \cdots, \omega_{k n_{k}}\right], k=1,2, \cdots, s
$$


Correspondingly, the process of stratified synthesis is performed by the following two steps.

The First Order Synthesis. Suppose the similarity vector of the $k^{\text {th }}$ group is

$$
\boldsymbol{R}_{k}=\left[\begin{array}{lll}
r_{1 k} & r_{2 k} & r_{3 k}
\end{array}\right]^{\mathrm{T}}
$$

Inserting Eqs. (6) and (7), the evaluation result of the $k t h$ index group is

$$
b_{k}=\boldsymbol{\omega}_{k} \cdot \boldsymbol{R}_{k}=\left[\omega_{k 1}, \omega_{k 2}, \cdots, \omega_{k n_{k}}\right]\left[\begin{array}{c}
r_{1 k} \\
r_{2 k} \\
r_{3 k}
\end{array}\right]
$$

The Second Order Synthesis. The second step is to synthesis the different groups. Inserting Eqs. (5) and (8), the evaluation result is

$$
E=\boldsymbol{\omega} \cdot \boldsymbol{B}=\left[\omega_{1}, \omega_{2}, \omega_{3}\right]\left[\begin{array}{l}
b_{1} \\
b_{2} \\
b_{3}
\end{array}\right]
$$

where $E$ is the integrated similarity between the target and the true warhead.

\section{Numerical example}

According to the proposed method, this section gives a simulation example. Suppose the target group consists of three targets, which are labeled as I, II, III, the bottom characteristic similarities are given in Table 2 .

Table 2. Bottom Similarity of Different Targets

\begin{tabular}{|l|r|r|r|}
\hline $\begin{array}{l}\text { Bottom } \\
\text { similarity }\end{array}$ & I & II & III \\
\hline Radiant intensity: $r_{11}$ & 0.80 & 0.85 & 0.30 \\
\hline Temperature: $r_{21}$ & 0.79 & 0.90 & 0.45 \\
\hline Image: $r_{31}$ & 0.85 & 0.88 & 0.29 \\
\hline RCS: $r_{12}$ & 0.88 & 0.80 & 0.85 \\
\hline Polarization: $r_{22}$ & 0.78 & 0.68 & 0.90 \\
\hline Image: $r_{32}$ & 0.85 & 0.71 & 0.92 \\
\hline Motion: $r_{13}$ & 0.90 & 0.50 & 0.60 \\
\hline Attitude: $r_{23}$ & 0.85 & 0.66 & 0.69 \\
\hline Fall point: $r_{33}$ & 0.80 & 0.70 & 0.66 \\
\hline
\end{tabular}

The integrated similarity can be calculated through the following step.

Determination of Index Weights. By consulting the specific specialist, the determinant matrixes of the three kind of index are defined as follows:

$$
A_{1}=\left[\begin{array}{ccc}
1 & 1 / 3 & 1 / 3 \\
3 & 1 & 1 \\
3 & 1 & 1
\end{array}\right], A_{2}=\left[\begin{array}{ccc}
1 & 1 / 3 & 1 \\
3 & 1 & 3 \\
1 & 1 / 3 & 1
\end{array}\right], A_{3}=\left[\begin{array}{ccc}
1 & 1 / 3 & 1 / 5 \\
3 & 1 & 1 / 2 \\
5 & 2 & 1
\end{array}\right]
$$

Then the solution of Eq. (2) can be obtained with

$$
\begin{aligned}
& \omega_{1}=[0.14,0.43,0.43] \\
& \omega_{2}=[0.2,0.6,0.2] \\
& \omega_{3}=[0.11,0.31,0.58]
\end{aligned}
$$

Meanwhile, the consistency indices of different matrixes are 


$$
\begin{aligned}
& C R_{1}=0 \\
& C R_{2}=0 \\
& C R_{3}=0.0032
\end{aligned}
$$

Determination of Index Group Weights. By consulting the specific specialist, the determinant matrix is

$$
A=\left[\begin{array}{ccc}
1 & 1 & 1 / 3 \\
1 & 1 & 1 / 3 \\
3 & 3 & 1
\end{array}\right]
$$

Substituting Eq. (2) yields

$$
\omega=[0.2,0.2,0.6]^{\mathrm{T}}
$$

Meanwhile, the consistency of the determinant matrix is

$$
C R=0
$$

The First Order Synthesis. According to Eq. (8), the results of the first order synthesis are

$$
\begin{aligned}
& b_{1 \mathrm{II}}=0.817, b_{2 \mathrm{I}}=0.814, b_{3 \mathrm{I}}=0.826 \\
& b_{1 \mathrm{II}}=0.884, b_{2 \mathrm{II}}=0.710, b_{3 \mathrm{II}}=0.666 \\
& b_{1 \mathrm{III}}=0.360, b_{2 \mathrm{III}}=0.894, b_{3 \mathrm{III}}=0.663
\end{aligned}
$$

The Second Order Synthesis. According to Eq. (9), the results of second order synthesis are

$$
\begin{aligned}
& E_{\mathrm{I}}=0.822 \\
& E_{\mathrm{II}}=0.718 \\
& E_{\mathrm{III}}=0.648
\end{aligned}
$$

As a result, the integrated similarities of the three targets have the order of $E_{I}>E_{I I}>E_{I I I}$. Therefore, target I has the highest probability to be the true warhead.

\section{Conclusion}

This paper proposed a stratified synthesis strategy to recognize the target. Firstly, the hierarchical index system of target characteristic similarity is established. Secondly, the AHP method is used to determine the index weight. Thirdly, stratified synthesis strategy is given to calculate the integrated similarity. Simulation shows that the proposed method gives an effective way to recognize the true warhead by considering multiple characteristics of the target.

The study proposed in this paper is just a preliminary research on the target recognition. There are still a lot of problems to be further investigated, such as the establishment of typical warhead library and the determination of bottom similarity. These are promising directions to be explored.

\section{References}

[1] M. Sessler, J. M. Cornwall, et al., Countermeasures: A Technical Evaluation of the Operational Effectiveness of the Planned US National Missile Defense System, Union of Concerned Scientists and MIT Security Studies Program, 2000.

[2] S. Z. Der, and R. Chellappa, 1997. Probe-based automatic target recognition in infrared imagery, IEEE Trans. Image Processing, 6(1): 92-102.

[3] H. Zhu, L. Z. Deng, G. Zhou, et al., 2012. Target recognition method based on template matching in downward-looking infrared image, presented at the International Conference on Audio, Language and Image Processing (ICALIP). 
[4] X. Sun, C. J. Zhu, D. S. Yang, et al., 2014. Research on infrared target recognition in complicated ground backdrop, presented at the International Conference on Progress in Informatics and Computing (PIC).

[5] M. Moruzzis, and N. Colin, 1998. Radar target recognition by fuzzy logic, IEEE AES Systems Magazine, pp. 13-19.

[6] K. Copsey, and A. Webb, 2003. Bayesian gamma mixture model approach to radar target recognition, IEEE Trans. Aerospace and Electronic Systems, 39(4): 1201-1217.

[7] F. Aldhubaib, N. V. Shuley, 2010. Radar target recognition based on modified characteristic polarization states, IEEE Trans. Aerospace and Electronic Systems, 46(4): 1921-1933.

[8] J. L. Dong, 2009. An improved fuzzy synthesis evaluation algorithm for software quality, presented at the Information Management, International Conference on Innovation Management and Industrial Engineering.

[9] W.Z. Xiao, Z. Y. Liu, and W. H. Zhong, 2011. Multi-level fuzzy synthesis evaluation on construction supply chain risk, presented at the Chinese Control and Decision Conference (CCDC).

[10] N. Yaraghi, P. Tabesh, P. Q. Guan, et al., 2015. Comparison of AHP and Monte Carlo AHP under different levels of uncertainty, IEEE Trans. Engineering Management, 62(1): 122-132.

[11] G. Zhang, L. X. Wang, A. P. Duffy, et al., 2015. Applying the analytic hierarchy process (AHP) to an FSV-based comparison of multiple datasets, IEEE Trans. Electromagnetic Compatibility, 57(3): 477-483. 